\title{
Geodynamic, geodetic, and seismic constraints favour deflated and dense-cored LLVPs
}

Fred D. Richards ${ }^{* 1,2}$, Mark J. Hoggard ${ }^{2,3,4}$, Sia Ghelichkhan ${ }^{4}$, Paula Koelemeijer ${ }^{5}$ \& Harriet C. P. Lau ${ }^{6}$

1. Department of Earth Science \& Engineering, Imperial College London, Royal School of Mines, Prince Consort Road, London, SW7 2AZ, UK

2. Department of Earth \& Planetary Sciences, Harvard University, 20 Oxford Street, Cambridge, MA, 02138, USA

3. Lamont-Doherty Earth Observatory, Columbia University, 61 Route 9W, Palisades, NY, 10964, USA

4. Research School of Earth Sciences, Australian National University, Building 142, Mills Road, Acton, ACT, 2601, Australia

5. Department of Earth Sciences, Royal Holloway, University of London, Egham Hill, Egham, Surrey, TW20 $0 \mathrm{EX}, \mathrm{UK}$

6. Department of Earth \& Planetary Science, University of California, Berkeley, 307 McCone Hall, Berkeley, CA, 94720, USA

*f.richards19@imperial.ac.uk

\begin{abstract}
Two continent-sized features in the deep mantle, the large low-velocity provinces (LLVPs), influence Earth's supercontinent cycles, mantle plume generation, and its geochemical budget. Seismological advances have steadily improved LLVP imaging, but several fundamental questions remain unanswered, including: What is their vertical extent? And, are they purely thermal anomalies, or are they also compositionally distinct? Here, we investigate these questions using a wide range of observations. The relationship between measured geoid anomalies and long-wavelength dynamic surface topography places an important upper limit on LLVP vertical extent of $\sim 900 \mathrm{~km}$ above the core-mantle boundary (CMB). Our mantle flow modelling suggests that anomalously dense material must exist at their base to simultaneously reproduce geoid, dynamic topography, and CMB ellipticity observations. We demonstrate that models incorporating this dense basal layer are consistent with independent measurements of semi-diurnal Earth tides and Stoneley modes. Our thermodynamic calculations indicate that a $\sim 100 \mathrm{~km}$-thick layer of early-formed, chondrite-enriched basalt is the chemical configuration most compatible with these geodynamic, geodetic and seismological constraints. By reconciling these disparate datasets for the first time, our results demonstrate that, although dominantly thermal structures, basal sections of LLVPs represent a primitive chemical reservoir that is periodically tapped by upwelling mantle plumes.
\end{abstract}

\section{Main}

Seismic tomographic models consistently image two large regions of slow seismic velocity in the deep mantle that are widely interpreted to be hotter than ambient material and are spatially correlated with positive, long-wavelength geoid height anomalies (Figures 1 and $2 \mathrm{a})^{1}$. Early mantle flow studies treated these features as buoyant upwellings and found that an increase in mantle viscosity with depth yields satisfactory model fits to observed non-hydrostatic 

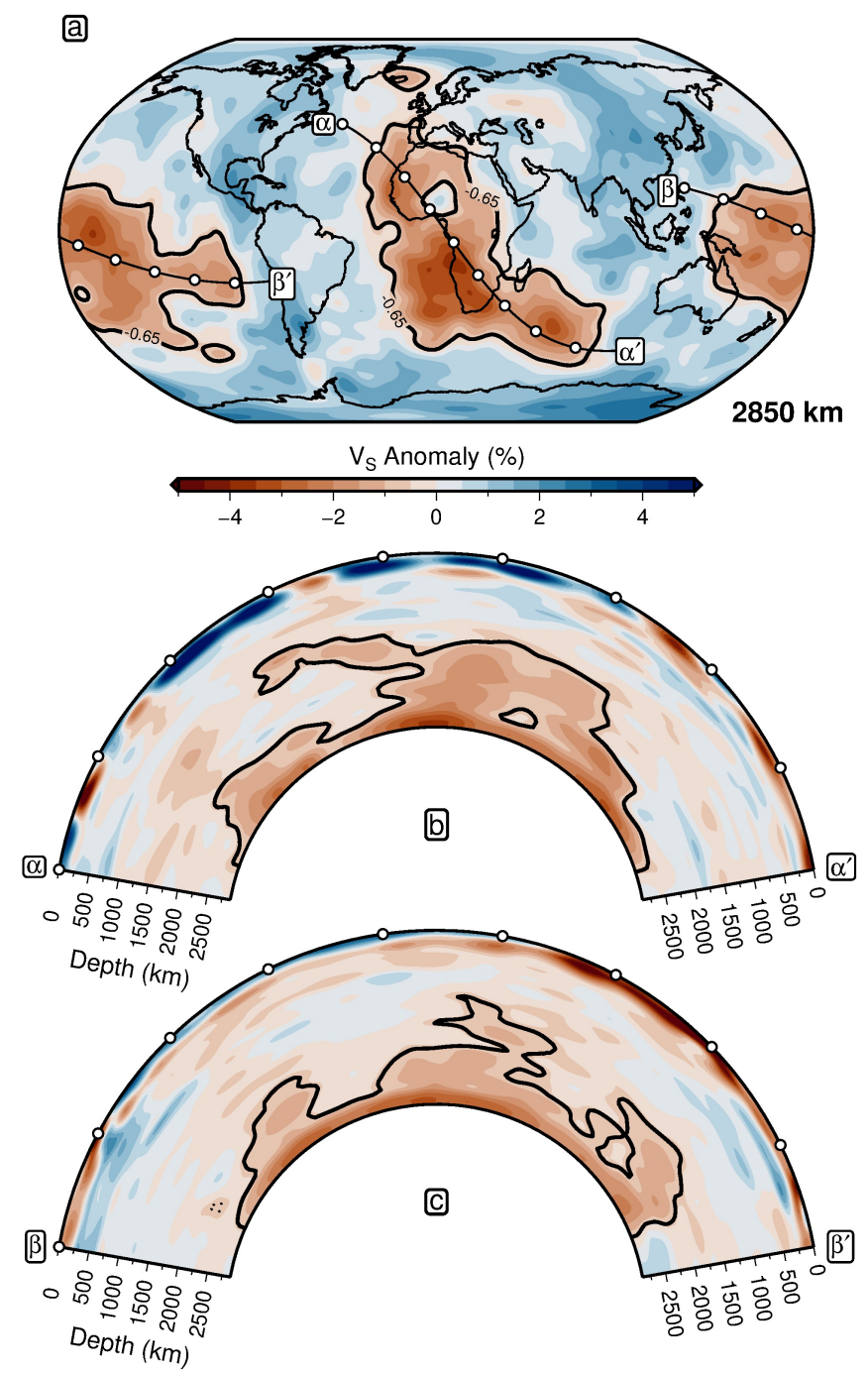

Figure 1: Spatial extent of seismically imaged LLVPs. (a) Shear-wave velocity $\left(V_{S}\right)$ anomalies at $2850 \mathrm{~km}$ depth in the TX2011 seismic tomographic model ${ }^{8}$. Thick black contour $=-0.65 \% V_{S}$ anomaly threshold used to delineate LLVP boundary ${ }^{9} ; \alpha-\alpha^{\prime}$ and $\beta-\beta^{\prime}=$ cross-section locations; white circles spaced at $1000 \mathrm{~km}$ intervals. (b) Cross-section $\alpha-\alpha^{\prime}$ beneath Africa through blended tomographic model (SLNAAFSA above $300 \mathrm{~km}$, TX2011 below $400 \mathrm{~km}$, linearly interpolated between 300-400 km). (c) Cross-section $\beta-\beta^{\prime}$ beneath Pacific Ocean.

geoid height anomalies ${ }^{2,3}$. Nevertheless, these instantaneous flow solutions are non-unique and suffer from tradeoffs between the magnitude and distribution of excess buoyancy. While there is now better agreement on the lateral extent of LLVPs ${ }^{4}$, numerous controversies remain concerning their structure and composition.

First, body wave coverage in the mid-to-lower mantle $(\sim 1000-2500 \mathrm{~km}$ depth $)$ is low and most ray paths that traverse this region are near-vertical, making global tomographic models susceptible to smearing artefacts in this depth range ${ }^{5,6}$. The vertical extent of LLVPs is therefore uncertain, with recent studies suggesting that laterally extensive low-velocity structures imaged at depths $\leq 2000 \mathrm{~km}$ may actually represent tomographic filtering of clusters of distinct plumes ${ }^{7}$.

Second, considerable debate remains over whether LLVPs are purely thermal or thermochemical features. Isotopic variations in intraplate volcanics ${ }^{10}$, joint seismic-geodynamic inversions ${ }^{11}$, body tides ${ }^{12}$, and their apparent stability with respect to the reconstructed locations of Phanerozoic kimberlites and large igneous provinces ${ }^{9}$, all suggest that LLVPs are enriched in chemically distinct and anomalously dense material. Numerical models suggest 
This preprint has been submitted to Nature Geoscience and is yet to undergo peer review.

that this material must have a $\sim 2-4 \%$ intrinsic chemical density excess to generate and preserve such compositional heterogeneity over billion-year timescales ${ }^{13,14,15}$. Seismic evidence in favour of chemically distinct LLVPs has, however, proven less conclusive. For example, the decorrelation between shear-wave velocity $\left(V_{S}\right)$ and bulk sound velocity $\left(V_{\phi}\right)$ below $2000 \mathrm{~km}$ depth has been inferred to support both thermal and thermochemical interpretations ${ }^{16,5}$. Similarly, strong lateral $V_{S}$ gradients at LLVP boundaries may point to chemical heterogeneity ${ }^{17}$, but several studies suggest that similar features may occur with purely thermal variations ${ }^{18,19,20}$. While normal mode studies generally prefer anomalously dense LLVPs ${ }^{21,16}$, recent Stoneley mode observations (i.e., normal modes trapped along the CMB) indicate that the LLVPs are, on average, positively buoyant, although a $\sim 100 \mathrm{~km}$-thick anomalously dense basal layer cannot be ruled out ${ }^{22}$. This result apparently contradicts inferences from body tide observations, which yield a mean excess density of $\sim 1 \%$ within the bottom $\sim 350 \mathrm{~km}$ of the $\operatorname{LLVPs}^{12}$.

While LLVP buoyancy structure remains uncertain, their morphology and the potential presence of chemically distinct material is expected to significantly influence spatiotemporal patterns of mantle circulation ${ }^{23,24,25,11}$. Since the early models of mantle flow ${ }^{2,3}$, there have been several important advances in geodynamic observables, notably improved present-day constraints on CMB excess ellipticity ${ }^{26}$ and the planform of surface dynamic topography ${ }^{27}$. Moreover, recent geodetic and seismological measurements of Earth's long-period motions-in particular, body tides and Stoneley modes - now provide additional bounds on deep mantle density structure. These developments allow us to investigate the trade-off between the magnitude and distribution of LLVP buoyancy, and to re-examine these controversies using new simulations of whole-mantle flow, tidal deformation and Stoneley mode oscillation. Using a suite of existing tomographic models, we perform geodynamic inversions to determine whether thermal or thermochemical density structures are more compatible with the geoid, CMB, and dynamic topography observations. The best-fitting density configurations are then tested against Stoneley mode splitting and body tide observations, and we demonstrate that the existing discrepancies between these datasets can be resolved. Finally, we explore geochemical implications of these inversion-derived buoyancy structures using thermodynamic calculations of the density and elastic properties of possible compositional endmembers. By analysing the fits of the resulting model predictions with a wide range of observations, we identify the nature and distribution of chemical heterogeneity within the deep Earth.

\section{Reconciling geodynamic observations and predictions}

Recent re-evaluation of dynamic surface topography using global inventories of residual depth measurements confirms that the long-wavelength component of this field is spatially correlated with geoid height anomalies (Figure 2ab) ${ }^{27,29}$. While there is some disagreement on the appropriate methodology for spectrally analysing these data, consensus has emerged for water-loaded amplitudes of $\pm 700 \mathrm{~m}$ at spherical harmonic degrees $l=1-3^{27,30,31,32,33}$. Meanwhile, geodetic observations of Earth's free core nutation place a narrow bound $(\sim 400 \pm 100 \mathrm{~m})$ on the amplitude of the degree-two $(l=2)$, order-zero $(m=0)$ component of non-hydrostatic CMB topography (i.e., excess ellipticity; Figure 2c) ${ }^{26}$. Unfortunately, efforts to map global CMB topography at shorter wavelengths using seismic data are presently hampered by trade-offs between velocity and density structure in the $\mathrm{D}^{\prime \prime}$ region ${ }^{34}$. 

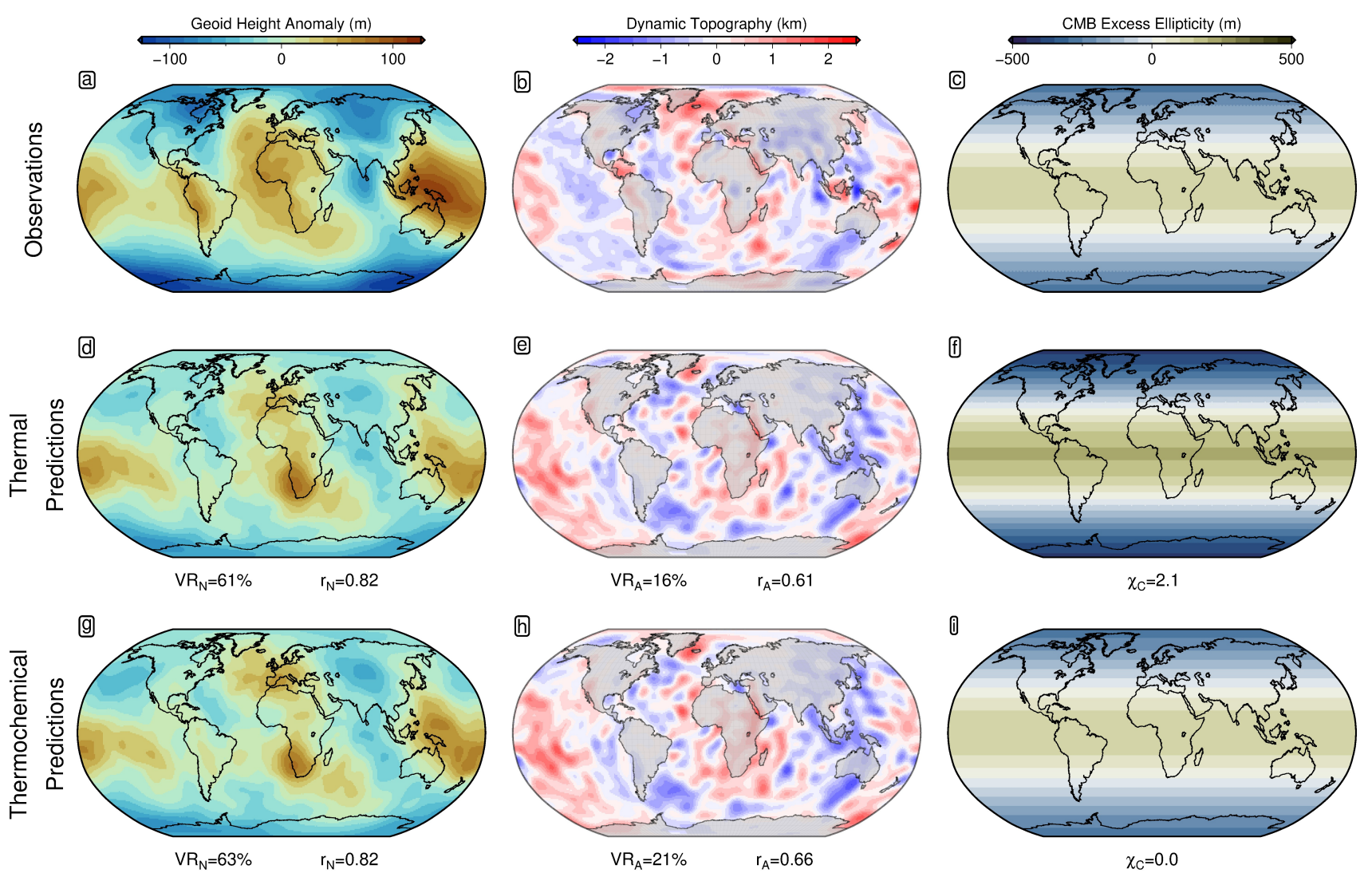

Figure 2: Observations versus optimal instantaneous flow modelling predictions for TX2011 tomographic model and S10 viscosity profile. (a) Observed non-hydrostatic geoid height anomalies ${ }^{28}$. (b) Observed dynamic surface topography ${ }^{29}$. (c) Observed excess CMB ellipticity ${ }^{26}$. (d) Predicted geoid for optimal mantle density model assuming LLVPs are purely thermal features. VR $=$ variance reduction; $r=$ Pearson's correlation coefficient (Methods). (e) Predicted dynamic topography for this model. (f) Predicted excess CMB ellipticity for this model. $\chi_{C}=$ misfit to observed CMB excess ellipticity (Methods). (g-i) Same for optimal density model that includes compositionally distinct LLVPs.

In light of these improved and revised constraints, we ask: Can a model of $V_{S}$-derived mantle density be constructed that simultaneously satisfies the geoid, dynamic topography, and excess CMB ellipticity? To investigate this issue, we have constructed a suite of $\sim 10^{6}$ density models, simulated the resulting instantaneous mantle flow, and computed misfits to the observational datasets (Methods). For the upper mantle above $400 \mathrm{~km}$, we have adopted a modified version of the RHGW20 density model ${ }^{35}$, which accounts for anelasticity at seismic frequencies and has been demonstrated to yield acceptable fits to short-wavelength dynamic topography. The deeper mantle is divided into five layers, and within each layer, the $V_{S}$-to-density scaling $\left(R_{\rho}=\frac{d \ln \rho}{d \ln V_{S}}\right)$ is varied between 0.1-0.4. This range is in line with expectations from mineral physics constraints on pyrolitic and mixed pyrolitic-basaltic compositions, which are both hypothetical compositions for an isochemical mantle ${ }^{36,11}$. To allow for limited seismic resolution and potential imaging artefacts in the lower mid-mantle $(1000-2000 \mathrm{~km})$, we also test $R_{\rho}=0$ in this region. In addition, we construct a suite of thermochemical models where chemical heterogeneity is represented as a density jump, ranging between $0.0-2.0 \%$, between the LLVP interior and exterior. We generate density models using five seismic tomographic models and perform instantaneous flow calculations using three mantle viscosity profiles (Methods).

Three key results emerge from this analysis. First, we find that acceptable fits to both the geoid and dynamic surface topography can be obtained for thermal and thermochemical density models (Table S1; Figures 2 and S1- 


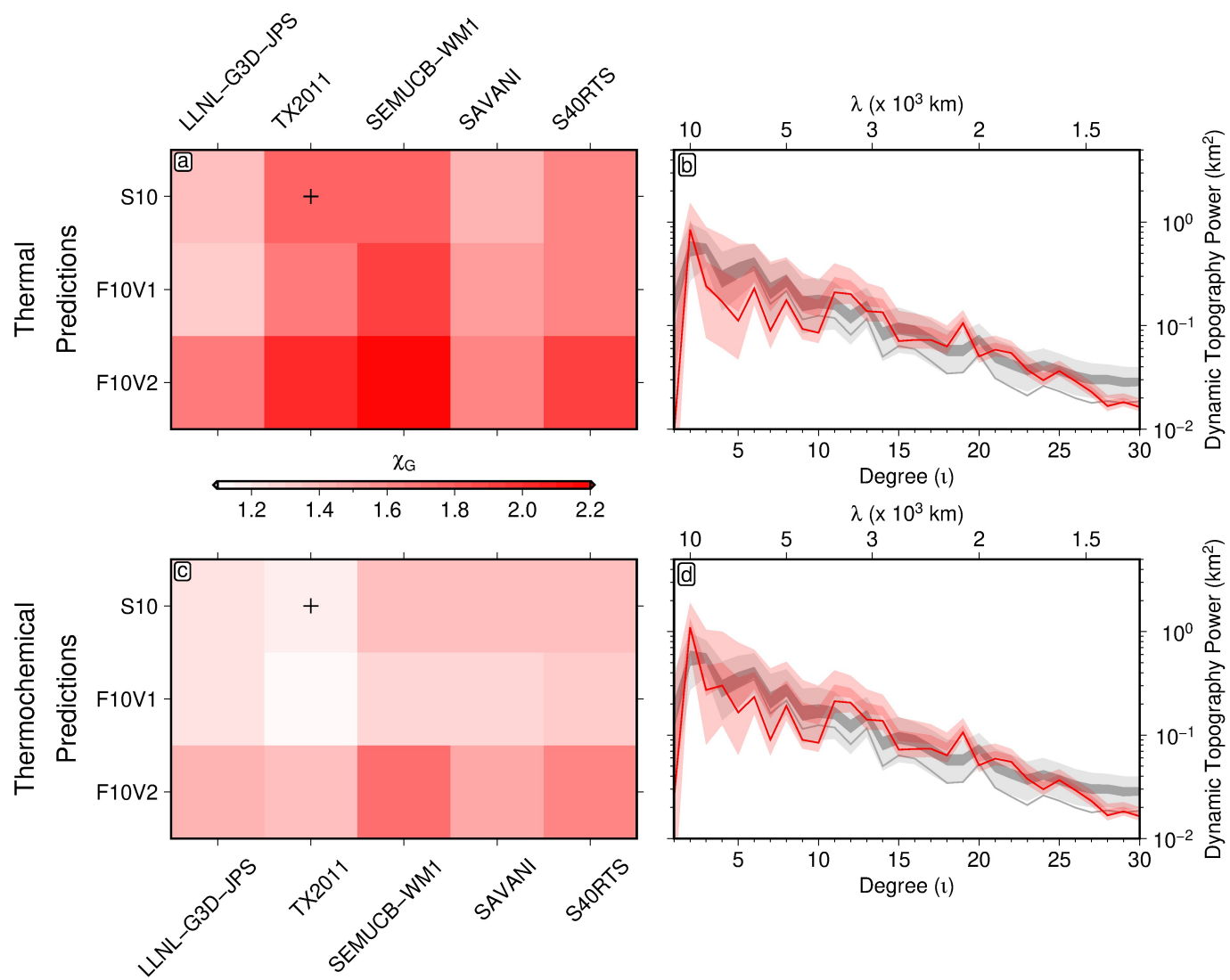

Figure 3: Geodynamic misfit as a function of input density and viscosity model. (a) Total geodynamic misfit, $\chi_{G}$ (Methods), of best-fit thermal models for each combination of viscosity and seismic tomographic input. Black cross = model shown in (b) and Figure 2d-f. (b) Observed and predicted dynamic topography power spectra of best-fit thermal model for TX2011 and S10 viscosity profile. Dark and light gray envelope $=99 \%$ and $50 \%$ confidence intervals for power spectrum of optimal spherical harmonic coefficients for oceanic residual depth measurements as constructed by Davies et al. ${ }^{32}$ using Automatic Relevance Determination algorithm (intervals derived from 100,000 random samples of inverted spherical harmonic coefficient probability distributions); solid gray line $=$ power spectrum of mean spherical harmonic coefficients determined for oceanic residual depth measurements; dark and light red envelope $=99 \%$ and $50 \%$ confidence intervals for power spectrum of thermal model constructed by sampling predicted dynamic topography at locations of shiptrack and point-wise oceanic residual depth measurements and determining optimal spherical harmonic coefficients using Gaussian Process algorithm of Valentine \& Davies ${ }^{37}$; solid red line = power spectrum of mean spherical harmonic coefficients determined for thermal model. (c) Total geodynamic misfit, $\chi_{G}$, of best-fit thermochemical models for each combination of viscosity and seismic tomographic input. Black cross = model shown in (d) and Figure 2g-i. (d) Observed and predicted dynamic topography power spectra of best-fit thermochemical model for TX2011 and S10 viscosity profile.

S6). Second, we obtain lower misfits and higher correlation coefficients and variance reductions for models that include compositionally distinct LLVPs relative to purely thermal models (Methods). This difference is particularly clear for the excess CMB ellipticity (Figure 2f versus 2i). Thermochemical models generally prefer strong excess density within the LLVP portion of the $\mathrm{D}^{\prime \prime}$ layer $\left(\delta \rho_{c} \geq+0.8 \%\right.$ for 13 of 15 tomographic and viscosity model combinations), but find little to no excess density in the $2000-2700 \mathrm{~km}$ depth range $\left(\delta \rho_{c} \leq+0.2 \%\right.$ for 13 of 15 models; Table S3; Figure S7). The thermochemical models also generally return $R_{\rho}$ values throughout the middle (400-1000 km) and lower (2000-2900 km) mantle that are in better agreement with experimental expectations for a pyrolitic composition ${ }^{36,11}$. Third, all best-fitting models require $R_{\rho} \sim 0$ for the 1000-2000 km mid-mantle layer, irrespective of whether or not LLVP regions are modelled as compositionally distinct (Tables S2-S3; Figure S7). 


\section{Vertical extent of LLVPs}

The geodynamic inversions exhibit a preference for $R_{\rho} \sim 0$ throughout the mid-mantle, which is too low for any plausible mantle compositions and indicates that geodynamic observables are incompatible with strong thermal buoyancy contributions from this depth. Given that seismic tomographic models are dominated by $l=2$ structure over the 1000-2000 km depth range, we explore this result further using associated sensitivity kernels for instantaneous mantle flow.

The geoid-to-topography amplitude ratio (GTR) at $l=2$ provides a crucial constraint on the vertical extent of LLVPs. In Figures $4 \mathrm{a}$ and $4 \mathrm{~b}$, we show the $l=2$ components of observed non-hydrostatic geoid height anomalies and water-loaded dynamic topography, which yield an estimated GTR of $\sim 0.21 \pm 0.07$. These deflections must be caused by $l=2$ density anomalies, with the strongest corresponding shear-wave velocity $\left(V_{S}\right)$ anomalies found within the LLVP regions, the mantle transition zone, and the asthenosphere (Figure 4e). These $V_{S}$ anomalies are anti-correlated with the observed geoid and dynamic topography, with the exception of the transition zone, where $V_{S}$ anomalies correlate with the geoid but remain anti-correlated or become decorrelated with dynamic topography (Figure $4 \mathrm{f}-\mathrm{g}$ ).
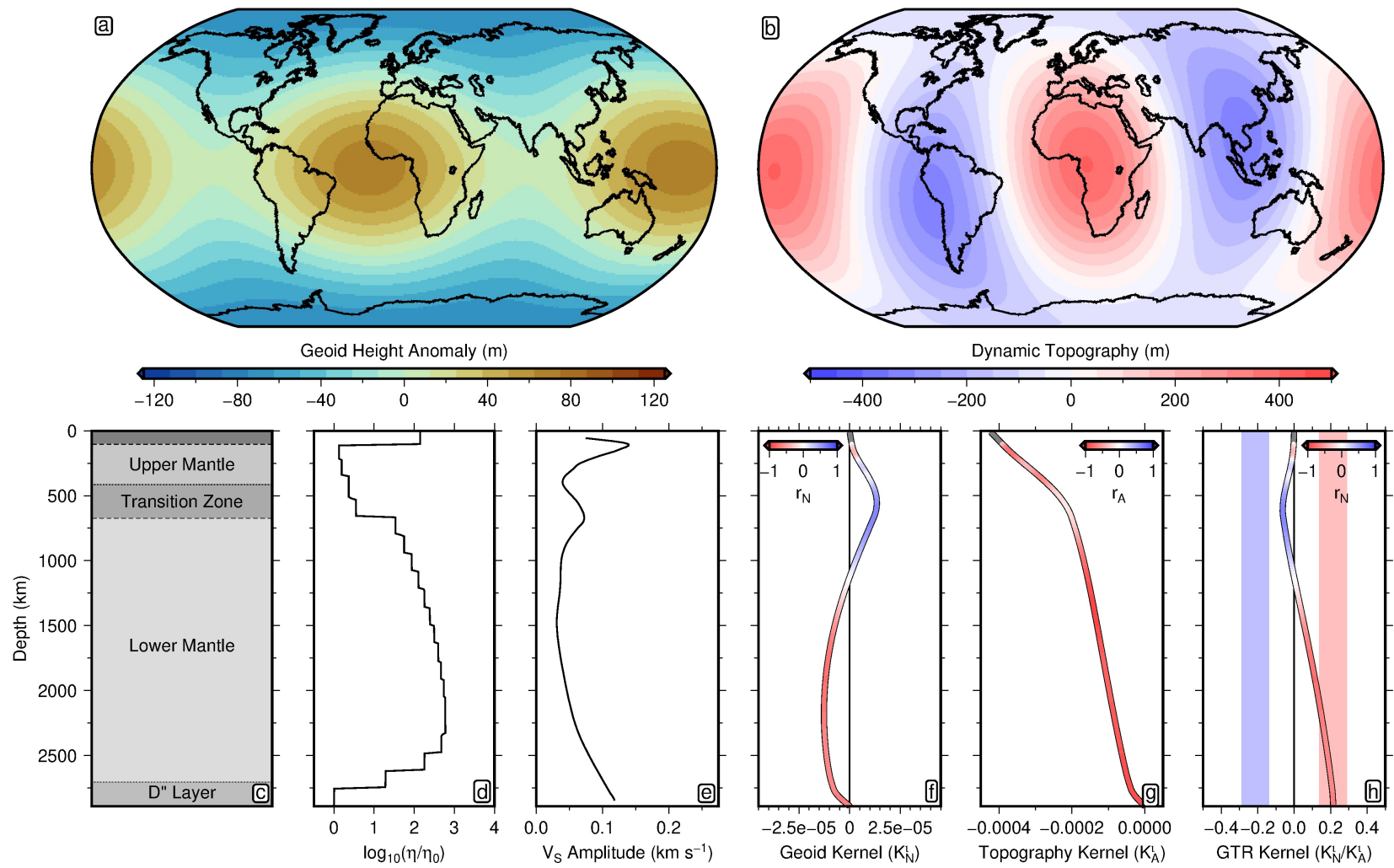

Dynamic Topography (m)
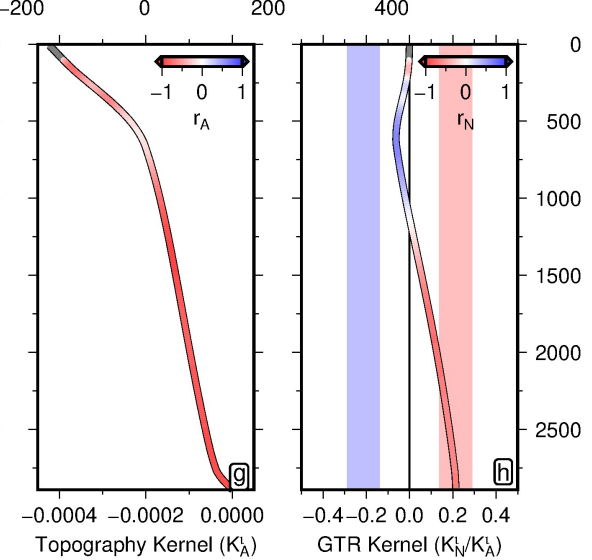

Figure 4: Relationship between long-wavelength $(l=2)$ dynamic topography, geoid and $V_{S}$ anomalies. (a) Observed non-hydrostatic geoid height anomalies ${ }^{28}$. (b) Observed water-loaded dynamic topography ${ }^{32}$. (c) Schematic radial mantle structure. (d) Normalised radial viscosity, $\eta$, profile $\left(\mathrm{S}_{10}{ }^{38}\right)$. (e) Spectral amplitude of $V_{S}$ anomalies from SEMUCB-WM1 tomographic model ${ }^{39}$. (f) Geoid kernel, $K_{N}^{l}$, coloured by geoid-to- $V_{S}$ anomaly correlation, $r_{N}$, as a function of depth. (g) Dynamic topography kernel, $K_{A}^{l}$, coloured by dynamic topography-to- $V_{S}$ anomaly correlation, $r_{A}$. (h) Geoid-to-topography ratio (GTR) kernel, coloured by $r_{N}$. Blue/red bands $=$ values required to produce the observed GTR when $V_{S}$ and thermal density anomalies are correlated/anti-correlated with the geoid.

The $l=2$ sensitivity kernels for the geoid, dynamic topography, and GTR (Figure 4f-h; Methods) are sensitive to the mantle viscosity profile (Figure $4 \mathrm{~d}$ ), but their shape is broadly consistent for a range of published profiles ${ }^{40,41}$ 
This preprint has been submitted to Nature Geoscience and is yet to undergo peer review.

(Figure S9). The $l=2$ GTR kernel shows that, to satisfy the observed value $(0.21 \pm 0.07)$, density anomalies must either anti-correlate with surface deflections in the deep mantle (intersection with red band, Figure 4h) or, in the transition zone, positively correlate with the geoid while remaining negatively correlated with dynamic topography (intersection with the blue band, Figure 4h). Our analyses reveal that deeper mantle structure is the major contributor to the integrated GTR, since this is the only region in which the predicted GTR kernel is consistent with observations. These kernels also show that any $l=2$, mid-mantle $(\sim 1000-2000 \mathrm{~km})$ thermal density anomalies can only lower the GTR. A mantle density model with LLVPs extending shallower than $\sim 2000 \mathrm{~km}$ depth that does fit the observed geoid will therefore simultaneously overpredict long-wavelength dynamic topography. Hence, the inversions return a preferred value of $R_{\rho} \approx 0$ in the mid-mantle. This finding provides strong evidence that LLVPs do not vertically extend beyond $900 \mathrm{~km}$ above the CMB, which is consistent with recent arguments that seismically imaged $l=2$, mid-mantle $V_{S}$ structure is an artefact of limited resolution ${ }^{7}$. Smaller scale density anomalies do exist in the 1000-2000 km depth interval (e.g., plumes and slabs ${ }^{39,42}$ ). However, instantaneous flow sensitivity kernels for shorter wavelengths approach zero over this depth range, such that these features are not expressed in the geoid, surface and CMB topography.

\section{Compatibility with body tides and Stoneley modes}

Despite similar sensitivity to deep Earth structure, previous studies based on semi-diurnal body tide and Stoneley mode splitting observations arrive at contrasting conclusions about LLVP density structure. The former show clear preference for the presence of anomalously dense material, with trade-offs between the amplitude and depth distribution of excess density ${ }^{12}$, while the latter prefer models with integrated density anomalies in the lower $400 \mathrm{~km}$ that are negative, as expected for a dominantly thermal control ${ }^{22}$. In light of these studies, we next test whether the mantle structure obtained from our optimal TX2011-based geodynamic models with thermochemical variations, or its purely thermal counterpart, is most consistent with these geodetic and seismological observations.

Goodness-of-fit to semi-diurnal body tide constraints is calculated following the methodology of Lau et al. ${ }^{12}$, which requires the improvement of predictions for 3D mantle structure over a $1 \mathrm{D}$ reference case to be significant at the 95\% level (Methods). The optimal TX2011-derived thermal model produces results that are only significant at the $93.8 \%$ level. By contrast, the best-fitting thermochemical density model based on the same tomographic input, but which include chemical heterogeneity in the base of LLVPs, yield statistically significant outcomes $(95.8 \%$ significance level).

We predict Stoneley mode splitting functions by adapting the methodology of Koelemeijer et al. ${ }^{22}$. Our revised approach has two methodological advantages over this study. Firstly, both the range and magnitude of $R_{\rho}$ tested here are consistent with candidate chemical compositions in the deep mantle ${ }^{11}$. Secondly, by calculating the instantaneous mantle flow associated with each model, CMB deflections are dynamically consistent with each LLVP density structure. We find that misfit between observed and predicted Stoneley mode splitting functions is $\sim 20 \%$ lower for the optimal TX2011-based thermochemical density model compared with its equivalent thermal model (Table S4; Figure S10). This conclusion appears to contradict the findings of Koelemeijer et al. ${ }^{22}$, and is 
This preprint has been submitted to Nature Geoscience and is yet to undergo peer review.

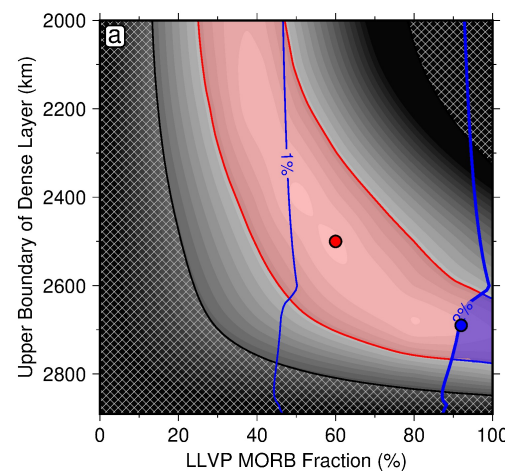
anomalies ${ }^{47,46}$.

partly explained by our methodological improvements and partly by the stronger $V_{S}$ amplitudes at $l=2$ below $2500 \mathrm{~km}$ depth in TX2011 compared to the SP12RTS model adopted in that study (Supplementary Information).

Significantly, these results indicate that the presence of anomalously dense material in the bottom $\sim 200 \mathrm{~km}$ of the LLVPs is not only compatible with available geodynamic constraints, but is also consistent with observations of Earth's semi-diurnal body tide and Stoneley mode splitting.

\section{Implications for lower mantle chemistry}

Having established that geodynamic, seismological and geodetic constraints provide evidence for the presence of a dense basal layer within the LLVPs, we explore the compatibility of different candidate compositions. Several hypotheses have been proposed for the formation of chemically distinct LLVP material, including: slow accumulation of basalt from subducted slabs reaching the $\mathrm{CMB}^{43}$; preservation of primordial mantle material segregated during top-down crystallisation of a basal magma ocean ${ }^{44}$; subduction of iron and silicon-rich Hadean crust along with a terrestrial regolith comprising chondritic and solarwind-implanted material ${ }^{45}$; and pooling of dense, iron-rich melts generated in the primordial mantle transition zone ${ }^{46}$.

We have assembled three endmembers to test the compositional range encompassed by these different scenarios: i) present-day mid-ocean ridge basalt (MORB; lowest $\mathrm{Fe}$, highest $\mathrm{Si}$ content) ${ }^{47}$; ii) chondrite-enriched Hadean basalt (intermediate $\mathrm{Fe}$ and $\mathrm{Si})^{45}$; iii) iron-enriched pyrolite (highest Fe, lowest $\mathrm{Si}$ ), representing early Archaean melts generated in the transition zone or remnants of a basal magma ocean ${ }^{46,44}$ (Table 1). For each of these compositions, we perform thermodynamic modelling ${ }^{48}$ and find that all options yield a positive density and negative shear-wave velocity contrast with respect to ambient pyrolitic mantle at deep mantle temperatures and pressures ( 2000-4000 K; 110-140 GPa; Figure S11). The amplitude of these contrasts vary, with modern basaltic material generating the weakest contrasts, while the most iron-rich primordial components produce the strongest
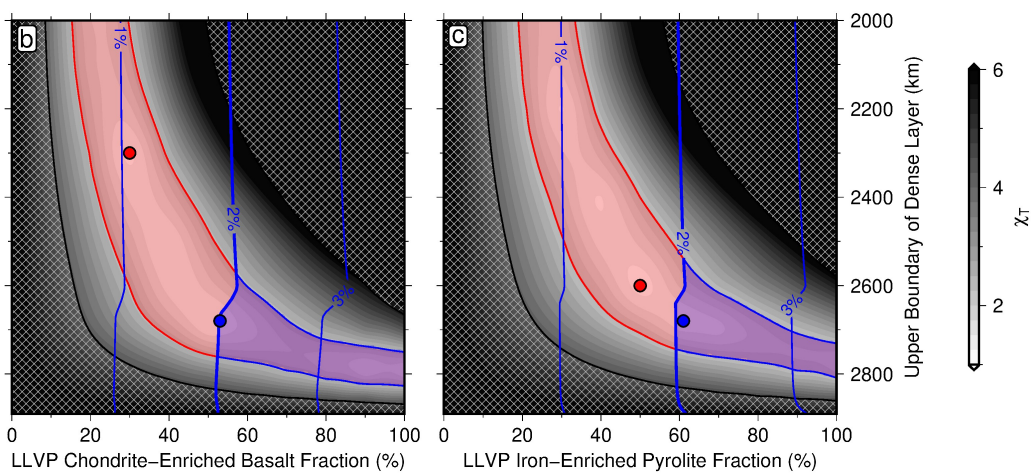

Figure 5: Combined misfit to geodynamic and Stoneley mode observations as a function of mantle composition. (a) Combined total misfit $\left(\chi_{T}\right)$ as a function of MORB ${ }^{47}$ fraction within LLVP. Material outside LLVP is assumed to be pyrolitic. Hatched region $=$ models with peak-to-valley $l=2 \mathrm{CMB}$ topography exceeding $\pm 4.7 \mathrm{~km}$ maximum constraint ${ }^{34} ;$ red circle $=$ best-fitting model; red shading $=$ models with misfit less than double that of global minimum; thin blue contours $=$ compositional density difference between dense layer material and ambient mantle; bold blue contour $=$ lower limit of suggested $\sim 2-4 \%$ compositional density threshold for long-term preservation of intra-LLVP chemical heterogeneity ${ }^{13,14,15}$; blue circle $=$ best-fitting model with intrinsic density anomaly above preservation threshold; blue shading = models with misfit less than double that of global minimum and compositional density anomaly above preservation threshold. (b) Same for primordial material (chondrite-enriched basalt ${ }^{45}$ ). (c) Same for primordial material (iron-enriched pyrolite ${ }^{46}$ ). 
This preprint has been submitted to Nature Geoscience and is yet to undergo peer review.

The relatively modest excess density below $2700 \mathrm{~km}$ recovered from our initial geodynamic inversions $\left(\overline{\delta \rho_{c}}=0.4-\right.$ $1.6 \%$ ) is consistent with mechanical mixtures comprising $20-70 \%$ pyrolite and $30-80 \%$ modern MORB, or 50-90\% pyrolite and $10-50 \%$ of either iron-rich primordial component. However, these excess densities fall below the $\sim 2-4 \%$ threshold required for long-term preservation of intra-LLVP chemical heterogeneity ${ }^{13,14,15}$. We therefore explore how a trade-off between the thickness of the basal layer and its excess density affects fits to the geodynamic and seismic constraints, and whether any of the proposed chemical compositions are more or less compatible. Instantaneous flow calculations are repeated with density models constructed from the thermodynamic predictions for different combinations of chemical components within and without the LLVPs (Methods). Mantle material is modelled as a mechanical mixture of pyrolite and each candidate composition, with density anomalies set to zero between 1000-2000 km depth based on the geodynamic inversion results. We find a strong trade-off between the anomalous density of the basal LLVP region and its thickness, with similar misfit to geodynamic observables obtained for thin, highly enriched versus thicker, less chemically distinct basal layers (Figure S16). Although results are dependent on the radial mantle viscosity profile, optimal fits are generally obtained for thinner, more enriched layers, irrespective of whether anomalously dense material within the LLVP is assumed to be basaltic or primordial. Best-fitting models for each chemical component yield similar misfit values, with optimal layer thicknesses of $\sim 200 \mathrm{~km}$. These basal layer configurations are consistent with geochemical constraints (based on tungsten isotopes in magmas) indicating intra-LLVP dense accumulations must be thin ${ }^{49}$.

Combining geodynamic and Stoneley mode misfit into a joint misfit function does not significantly reduce the trade-off between basal layer thickness and density (Methods; Figures S13-S16 and S18-S19). Nevertheless, while each endmember composition can generate densities satisfying the $2-4 \%$ excess density threshold for longterm chemical heterogeneity preservation ${ }^{13,14,15}$, the two primordial candidates yield a $\sim 10 \%$ reduction in joint misfit to Stoneley mode and geodynamic observations compared with recycled MORB (Figure 5a-c). The optimal chondrite-enriched basaltic model gives $\sim 5 \%$ lower misfit than its iron-enriched pyrolitic counterpart, indicating that a 100-200 km-thick layer mainly composed of sequestered, Hadean oceanic crust is most consistent with available data. The elevated $\mathrm{SiO}_{2}$ content of this basaltic composition also helps to explain the observed spatial decorrelation between $V_{\phi}$ and $V_{S}$ in the lowermost mantle, provided bridgmanite is at least partially replaced by post-perovskite within this depth range ${ }^{50,5}$ (Figure S11). Finally, the less extreme reduction in $V_{S}$ at lowermost mantle conditions for primordial basalt, compared to iron-enriched pyrolite, is more compatible with the relatively modest $V_{S}$ gradients that have been inferred across LLVP boundaries ${ }^{19,20}$. Consequently, we conclude that the available geodynamic, geodetic and seismological constraints on deep mantle structure are most compatible with LLVPs that have a vertical extent $\leq 900 \mathrm{~km}$ and a 100-200 km-thick basal layer composed primarily of Hadean, chondrite-enriched basaltic material.

Our preferred density model, characterised by muted long-wavelength mid-mantle structure and chemical heterogeneity concentrated in the deepest 100-200 km of the LLVPs, has important implications for mantle evolution, reducing the amplitude and slowing the rate of change of surface dynamic topography. By adopting this structure and validating its associated mantle flow field against evidence for continent-scale uplift and subsidence encoded in the geological record, our understanding of Earth's internal dynamics can be greatly refined, allowing impacts 
on landscape evolution and palaeoclimatic shifts to be determined with unprecedented fidelity.

\section{Acknowledgments}

F.D.R. acknowledges support from the Imperial College Research Fellowship Scheme and the Schmidt Science Fellows program, in partnership with the Rhodes Trust. M.J.H. acknowledges support from the National Aeronautics and Space Administration (grant NNX17AE17G), the Donors of the American Chemical Society Petroleum Research Fund (59062-DNI8), and Geoscience Australia. M.J.H. and S.G. are supported by the G-ADOPT project, which is funded by the Australian Research Data Commons (https://doi.org/10.47486/PL031). P.K. acknowledges support from a Royal Society University Research Fellowship (URF $\backslash$ R1 $\backslash 180377)$. H.C.P.L. acknowledges support from NSF (EAR-192386). We thank D. Al-Attar, J. Austermann, L. Colli, S. Cottaar, D. R. Davies, R. Hawkins, J. Mitrovica, M. Sambridge, Y. Takei, A. Valentine, N. White and J. Winterbourne for their help. Figures were prepared using Generic Mapping Tools software.

\section{Author Contributions}

F.D.R. and M.J.H. conceived this study. F.D.R. designed, set up and processed geodynamical simulations and relevant input models in consultation with M.J.H. S.G. wrote software for computing instantaneous mantle flow. S.G. and M.J.H. developed tools for constructing anelasticity-corrected input density models from thermodynamic lookup tables. P.K. developed the computing infrastructure used to calculate predicted Stoneley mode splitting functions. H.C.P.L. developed the software to compute body tide responses and conducted statistical analysis of the outputs. F.D.R. and M.J.H. integrated interdisciplinary components. F.D.R. compiled the supplementary information and wrote the manuscript with M.J.H., following discussions with and contributions from all authors.

Competing Interests: The authors declare no competing interests.

\section{Methods}

\section{Mantle Density Models}

We develop two classes of density models; the first based on inversion of geodynamic data, the second derived using thermodynamic forward modelling of proposed deep mantle compositions. To generate the first class of density models, we separate the mantle into six layers: 0-400 km (UUM = upper upper mantle), 400-670 km (LUM = lower upper mantle), 670-1000 km (UMM = upper mid-mantle), 1000-2000 km (LMM = lower mid-mantle), 2000$2700 \mathrm{~km}$ (ULM = upper lower mantle), and 2700-2891 km (LLM = lower lower mantle). Density in the UMM layer is determined from SLNAAFSA ${ }^{51}$, which is a version of the SL2013sv ${ }^{52}$ upper mantle model into which the regional updates SL2013NA in North America ${ }^{53}$, AF2019 in Africa ${ }^{54}$, and SA2019 in South America and the South 
This preprint has been submitted to Nature Geoscience and is yet to undergo peer review.

Atlantic Ocean ${ }^{55}$ have been incorporated. The baseline model, SL2013sv, has been shown to produce topographic predictions that are in good agreement with residual depth measurements, even at relatively short wavelengths $(\sim 1000 \mathrm{~km})^{35}$.

Seismic velocities are converted into density within the UMM layer using an anelastic parameterisation following the methodology of Richards et al. ${ }^{35}$. This approach allows self-consistent mapping between seismic velocities and temperature, density and viscosity variations, while correcting for discrepancies between tomographic models that result from parameterisation choices rather than true Earth structure. Optimal parameters determined for SLNAAFSA are: $\mu_{0}=75.9 \mathrm{GPa} ; \frac{\partial \mu}{\partial T}=-17.9 \mathrm{MPa}{ }^{\circ} \mathrm{C}^{-1} ; \frac{\partial \mu}{\partial P}=2.54 ; \eta_{r}=10^{23.0} \mathrm{~Pa} \mathrm{~s} ; E_{a}=489 \mathrm{~kJ} \mathrm{~mol}^{-1}$; $V_{a}=0.63 \mathrm{~cm}^{3} \mathrm{~mol}^{-1}$; and $\frac{\partial T_{s}}{\partial z}=0.931{ }^{\circ} \mathrm{C} \mathrm{km}^{-1}$. We assume that continental lithosphere, delineated by the $T=1200^{\circ} \mathrm{C}$ isothermal surface, has neutral buoyancy and set density in these regions equal to the average density of all external material at the relevant depth to eliminate any direct dynamic topographic contribution. This assumption is based on heat flow measurements, xenolith geochemistry, seismic velocity, gravity, and topography observations that suggest compositional and thermal density contributions approximately balance each other within the continental lithosphere ${ }^{56,57}$.

Below $300 \mathrm{~km}$, seismic velocity perturbations from a range of whole-mantle tomographic models (LLNL-G3D-

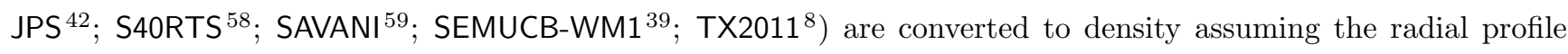
of PREM ${ }^{60}$ and constant $R_{\rho}=\partial \ln \rho / \partial \ln V_{S}$ values within each layer. To ensure smooth transitions in density anomalies between the two input density parameterisations, we take their weighted average between $300 \mathrm{~km}$ and $400 \mathrm{~km}$, beyond which the sensitivity of the surface wave-dominated upper mantle model tends to zero. Weighting coefficients of the respective tomographic models, $w_{U M}$ and $w_{W M}$, vary linearly between 1 and 0 over this depth range and are combined according to $w_{U M}=1-w_{W M} . R_{\rho}$ is fixed at 0.15 for the whole-mantle model between 300-400 km, based on the mean value within this layer inferred from SLNAAFSA.

The lower mantle layers, ULM and LLM, are laterally subdivided into regions outside (OULM and OLLM), and within the LLVPs (LULM and LLLM), each delineated using the $-0.65 \% V_{S}$ anomaly contour of the whole-mantle tomographic model under investigation ${ }^{9}$. Outside the LLVPs, $R_{\rho}$ varies as $R_{\rho}=[0.1,0.2, \ldots 0.4]$ with the exception of the LMM layer (1000-2000 km), where a minimum bound on $R_{\rho}$ of 0.0 is adopted allowing for limited mid-mantle seismic resolution and the potential presence of artefacts due to vertical smearing. Within the LLVPs, we apply a constant compositional density anomaly such that $\delta \rho(z)=R_{\rho}(i) \delta V_{S}(z)+\delta \rho_{c}(i)$, where $\delta \rho_{c}(i)$ is the intrinsic compositional density difference between LLVP material and ambient mantle ( $z$ is depth, $i$ is the layer index, i.e., ULM or LLM). Note that, in contrast to studies that employ negative $R_{\rho}$ values ${ }^{16,22,12}$, this approach maximises intra-LLVP density around the edges of the low-velocity regions rather than their central portions, and therefore assumes that, within each domain, internal $V_{S}$ variations are dominantly controlled by temperature rather than composition (Figure S8). This configuration is therefore consistent with the hypothesis that sharp compositional contrasts are responsible for strong lateral gradients in $V_{S}$ across the LLVP boundaries ${ }^{17}$. For our models, $\delta \rho_{c}$ varies as $[0 ., 0.2, . ., 2.0] \%$ within the LULM and LLLM regions, yielding $\sim 2 \times 10^{5}$ input density structures.

The second class of density models are created to investigate likely chemical compositions of the LLVPs. We generate a suite of density structures based on thermodynamic modelling of key candidate compositions and $V_{S}$ 
This preprint has been submitted to Nature Geoscience and is yet to undergo peer review.

variation from the tomographic model that produced optimal agreement with geodynamic observables, TX2011 (Table 1).

$\begin{array}{cccccccc}\text { Composition } & \mathrm{SiO}_{2}(\%) & \mathrm{MgO}(\%) & \mathrm{FeO}(\%) & \mathrm{CaO}(\%) & \mathrm{Al}_{2} \mathrm{O}_{3}(\%) & \mathrm{Na}_{2} \mathrm{O}(\%) & \mathrm{Reference} \\ \text { Pyrolite } & 38.71 & 49.85 & 6.17 & 2.94 & 2.22 & 0.11 & 47 \\ \text { MORB } & 51.75 & 14.94 & 7.06 & 13.88 & 10.19 & 2.18 & 47 \\ \text { CEB } & 48.47 & 20.00 & 11.28 & 10.59 & 8.16 & 1.50 & 45 \\ \text { FSP } & 40.15 & 41.98 & 12.90 & 2.82 & 1.92 & 0.23 & 46\end{array}$

Table 1: Molar oxide ratios for different mantle compositional endmembers. MORB = present-day mid-ocean ridge basalt; $\mathrm{CEB}=$ chondrite-enriched basalt; FSP = iron-enriched pyrolite.

For a given composition, Perple_X is used alongside the thermodynamic database of Stixrude \& LithgowBertelloni ${ }^{61}$ to generate a lookup table of anharmonic shear-wave velocities and densities varying temperature by $[300,350, . .4500] \mathrm{K}$ and pressure by $[0 ., 0.1, \ldots 140] \mathrm{GPa}$. At each depth, temperature-dependent discontinuities in density and seismic velocity caused by phase transitions are smoothed by adopting the median temperature derivative across a $\pm 500^{\circ} \mathrm{C}$ swath either side of the geotherm. Smoothed anharmonic velocities are then corrected for anelasticity using a $Q$ profile determined using the approach of Matas \& Bukowinski ${ }^{62}$, as outlined in Lu et al. ${ }^{11}$ (Figure S12). Having smoothed and corrected the $V_{S}$ lookup table, velocities from a given seismic tomographic model can be converted into temperature at each depth, with values adjusted by a constant offset to ensure mean temperatures are consistent with the geotherm. These temperatures are then used to extract the corresponding buoyancy structure from smoothed density lookup table. In cases where compositions are not equivalent to a particular endmember, properties appropriate for a mechanical mixture of the two components are calculated using the Voigt-Reuss-Hill approximation to average the elastic moduli. When the composition of the LLVP is distinct from ambient mantle, temperatures and densities are determined separately for the two components and then combined into a single array, with the boundary corresponding to the $-0.65 \% V_{S}$ anomaly contour ${ }^{9}$. All models assume that the range of possible mantle compositions is some combination of pyrolite and a specific dense component; either mid-ocean ridge basalt ${ }^{47}$, chondrite-enriched basalt ${ }^{45}$, or iron-enriched pyrolite ${ }^{46}$. For each component, we generate models for compositional enrichments of $[0,10, \ldots, 100] \%$ and for upper boundaries of the dense layer between $2000 \mathrm{~km}$ and $2800 \mathrm{~km}$ in $100 \mathrm{~km}$ increments, as well as $2850 \mathrm{~km}$ and the CMB (2890 km).

In the upper $300 \mathrm{~km}$, these density models are identical to those constructed using $R_{\rho}$ and $\delta \rho_{c}$ values; below $400 \mathrm{~km}$, densities are taken directly from the thermodynamically self-consistent parameterisation described above; and between $300 \mathrm{~km}$ and $400 \mathrm{~km}$ depth, densities derived from the two parameterisations are smoothly merged by taking their weighted average, as described for the first class of models. Since optimal thermal and thermochemical density models recovered from geodynamic inversions consistently find that $R_{\rho}(\mathrm{LMM}) \sim 0$, density anomalies in the 1000-2000 km depth interval are set to zero for all models (Figure S17).

\section{Mantle Flow Simulations}

Using the suite of thermal and thermochemical mantle density models, we predict surface and CMB dynamic topography and geoid undulations for $1 \leq l \leq 30$ using an instantaneous flow kernel methodology. As Earth's viscosity structure is uncertain, we assess the sensitivity of our mantle flow results to three different radial profiles 
This preprint has been submitted to Nature Geoscience and is yet to undergo peer review.

that are constrained by geoid, heat flow and glacial isostatic adjustment observations: S10 ${ }^{38}$; F10V1 ${ }^{40}$; F10V2 ${ }^{40}$.

To calculate instantaneous mantle flow, we exploit the sensitivity kernel methodology originally implemented by Hager \& O'Connell ${ }^{63}$ and Richards \& Hager ${ }^{64}$, extended to account for compressibility and self-gravitation ${ }^{65}$. This approach applies the propagator matrix technique to solve the equations governing conservation of mass and momentum within a highly viscous spherical shell, alongside Poisson's equation, to generate kernels describing the linear relationship between geodynamic observables (dynamic topography, geoid and CMB topography) and laterally varying density anomalies across the mantle. We impose free-slip surface and CMB boundary conditions. The resulting sensitivity kernels vary as a function of depth, the assumed viscosity profile, and the spherical harmonic degree under consideration. Dynamic topography $\delta A^{l m}$ can then be determined using

$$
\delta A^{l m}=\frac{1}{\Delta \rho_{0}} \int_{R_{C}}^{R_{\oplus}} K_{A}^{l}(r) \delta \rho^{l m}(r) d r
$$

where $K_{A}^{l}$ is the dynamic topography kernel, $r$ is radius, $\Delta \rho_{0}$ is the density difference between the uppermost mantle $\left(\rho_{0}=3380 \mathrm{~kg} \mathrm{~m}^{-360}\right)$ and water $\left(\rho_{w}=1030 \mathrm{~kg} \mathrm{~m}^{-3}\right), l$ and $m$ are spherical harmonic degree and order, $R_{\oplus}=6371 \mathrm{~km}$ and $R_{C}=3480 \mathrm{~km}$ are the radii of the Earth and CMB, respectively, and $\delta \rho^{l m}(r)$ represents the driving density anomalies. The geoid, $\delta N^{l m}$, is calculated using

$$
\delta N^{l m}=\frac{4 \pi \gamma R_{\oplus}}{(2 l+1) g_{R_{\oplus}}} \int_{R_{C}}^{R_{\oplus}} K_{N}^{l}(r) \delta \rho^{l m}(r) d r
$$

where $K_{N}^{l}$ is the geoid kernel, $g_{R_{\oplus}}$ is surface gravity and $\gamma$ is the gravitational constant. CMB topography, $\delta C^{l m}$, is determined according to

$$
\delta C^{l m}=-\frac{1}{\Delta \rho_{C}} \int_{R_{C}}^{R_{\oplus}} K_{C}^{l}(r) \delta \rho^{l m}(r) d r
$$

where $K_{C}^{l}$ is the CMB topography kernel and $\Delta \rho_{C}$ is the density difference between the lowermost mantle $\left(\rho_{C}=5570 \mathrm{~kg} \mathrm{~m}^{-3}\right)$ and the uppermost outer core $\left(\rho_{O C}=9900 \mathrm{~kg} \mathrm{~m}^{-3}\right)^{60}$.

Applying this kernel formalism permits rapid calculation of key observables, enabling the more complete exploration of parameter space central to this study. This method, however, cannot incorporate lateral viscosity variations (LVVs). While LVVs are undoubtedly present within the Earth, numerous studies conclude that they generate minimal differences in the geodynamical observations we explore here compared with those resulting from variability in density inputs derived from different tomographic models ${ }^{66,67,30}$. We therefore anticipate that our main conclusions remain valid for reasonable amplitudes of LVV.

\section{Misfit to geodynamic observations}

We assess model performance using a combined misfit function to assess compatibility with geoid, dynamic topography and excess CMB ellipticity constraints. Following previous studies ${ }^{68,69}$, we define the misfit to geoid and dynamic topography based on variance reduction (VR), a proxy for the proportion of observed signal explained by a given model prediction. Geoid misfit, $\chi_{N}$, is defined to be equivalent to $1-\mathrm{VR}_{N}$, where $\mathrm{VR}_{N}$ represents geoid 
variance reduction, and is calculated globally using

$$
\chi_{N}=\frac{\sum_{l=2}^{l_{\max }} \sum_{m=-l}^{l}\left(N_{c}^{l m}-N_{o}^{l m}\right)^{2}}{\sum_{l=2}^{l_{\max }} \sum_{m=-l}^{l}\left(N_{o}^{l m}\right)^{2}}
$$

where $N^{l m}$ terms represent spherical harmonic coefficients of observed (subscript $o$ ) and predicted (subscript $c$ ) geoid, and $l_{\max }=30$ is the maximum spherical harmonic degree. Dynamic topography misfit, $\chi_{A}$, is defined analogously to $\chi_{N}$ (i.e., $\chi_{A}=1-\mathrm{VR}_{A}$ ). However, since accurate residual depth measurements only exist at specific oceanic locations, rather than compare spherical harmonic coefficients, we instead determine this value in the spatial domain according to

$$
\chi_{A}=\frac{\sum_{n_{A}=1}^{N_{A}}\left[\left(A_{c}^{i}-A_{o}^{i}\right)-\overline{\left(A_{c}^{i}-A_{o}^{i}\right)}\right]^{2}}{\sum_{n_{A}=1}^{N_{A}}\left(A_{o}^{i}-\overline{A_{o}^{i}}\right)^{2}},
$$

where $A^{i}$ terms are predicted and observed dynamic topography at $N_{A}=2278$ geographic locations ${ }^{29}$, and values are weighted by the surface area of the $1^{\circ}$ bin associated with each data point in order to correct for latitudinal variation in sampling density. Since excess CMB ellipticity is defined using a single spherical harmonic coefficient, rather than using a variance reduction-based misfit definition, we use the expression

$$
\chi_{C}=\sqrt{\left(\frac{C_{c}^{20}-C_{o}^{20}}{\sigma_{C_{o}^{20}}}\right)^{2}}
$$

for this component, which is similar to previous studies ${ }^{70,69}$. $C^{20}$ terms represent the $l=2, m=0$ coefficient of observed and modelled core-mantle boundary topography, and $\sigma_{C_{o}^{20}}=100 \mathrm{~m}$ based on the range of reported values $^{71,72,26}$. Finally, we sum each of these three components into a combined geodynamic misfit function,

$$
\chi_{G}=\chi_{N}+\chi_{A}+\chi_{C} .
$$

In Figure 2, we present optimal results for the S10 viscosity profile ${ }^{38}$ and TX2011 tomographic model ${ }^{8}$ (Figures S1-S6 display results for other combinations). We select this tomographic model as it generates geodynamic predictions with the lowest overall misfit, while $\mathrm{S} 10^{38}$ is chosen over the $\mathrm{F} 10 \mathrm{~V} 1^{40}$ viscosity profile — despite the latter yielding lower misfits - since it does not include a very low viscosity $\left(7 \times 10^{19} \mathrm{~Pa}\right.$ s $)$ layer at the base of the transition zone, which has generally been considered controversial since it requires the entire region to be nearly water-saturated $(\sim 1.5 \%)^{73}$.

\section{Body tide and Stoneley mode predictions}

Modelling of the Earth's body tidal response requires models of 3D elastic, 3D density, and 1D anelastic structure. In the upper $400 \mathrm{~km}$ of the mantle, 3D elastic structure is determined using the calibrated parameterisation of SLNAAFSA to remove anelastic reductions in $V_{S}$ from the seismic tomographic model, leaving only anharmonic $V_{S}$ variations $\left(V_{S}^{a n h}\right)$. Below $300 \mathrm{~km}, V_{S}^{a n h}$ is derived from the tomographic values, $V_{S}^{a n e l}$, using radial changes in shear attenuation, $Q_{S}^{-1}$, from PREM and the expression $V_{S}^{a n e l}=V_{S}^{a n h}\left[1-\frac{Q_{S}^{-1}}{2 \tan (\pi \alpha / 2)}\right]$, where $\alpha=0.15^{60,74,75}$. 
This preprint has been submitted to Nature Geoscience and is yet to undergo peer review.

While the resulting 3D $V_{S}^{a n h}$ model constrains the unrelaxed shear modulus, unrelaxed bulk modulus variations are obtained from $V_{\phi}^{a n h}$, assuming that $R_{b}=\partial \ln V_{\phi} / \partial \ln V_{S} \sim \partial \ln V_{\phi}^{a n h} / \partial \ln V_{S}^{a n h}=0.05$ and the radial $V_{\phi}^{a n h}$ profile can be determined using the same $V_{S}-V_{\phi}$ scaling as PREM ${ }^{60}$. The 1D anelastic structure applied to determine elastic modulus dispersion at the 12-hour period of the M2 body tide adopts the mean value of $Q_{S}^{-1}$ obtained from the calibrated parameterisation of SLNAAFSA at depths above $400 \mathrm{~km}$, and that of PREM at greater depths.

With the Earth model specified, the body tide response is computed using full-coupling normal mode perturbation theory, with shear and bulk moduli dispersion at tidal frequencies using IERS standards ${ }^{75,76}$. Following Lau et $a l .{ }^{12}$, the fit between the predicted and observed in-phase M2 body tide displacement is assessed at GPS stations by determining whether inclusion of 3D elastic and density structure significantly enhances coherence between the two fields compared with a baseline 1D model $\left(\mathrm{PREM}^{60}\right)$. The 3D Earth model is only considered to yield a statistically significant improvement if the correlation obtained between 'raw' and 'corrected' GPS residuals exceeds that obtained for the 1D model at the 95\% significance level, accounting for correlation between GPS estimates due to the uneven spatial distribution of receivers. Raw residuals represent observed M2 body tide displacements minus those predicted for the 1D model. Corrected residuals also account for the effects of Moho and CMB excess ellipticity, Earth rotation and ocean tidal loading, and, in the 3D model case, incorporate an additional correction for differences in the body tide displacement predicted using 3D versus 1D structure.

To predict Stoneley mode splitting functions, 3D variations in $V_{S}$, compressional-wave velocity $\left(V_{P}\right)$ and CMB topography must be specified in addition to the density model ${ }^{77}$. $V_{S}$ anomalies are drawn directly from the tomographic model used to construct a given density model, while $V_{P}$ is determined by scaling $V_{S}$ anomalies using a constant value of $R_{P}=\partial \ln V_{P} / \partial \ln V_{S}=0.5^{78}$. We do not consider seismic anisotropy. CMB topography is determined self-consistently using instantaneous flow simulations that incorporate each density model.

For a specified input velocity, density and topography model, Stoneley mode splitting coefficients, $\mathcal{C}^{s t}$ can be calculated using the expression

$$
\mathcal{C}^{s t}=\int_{R_{C}}^{R_{\oplus}} \operatorname{dln} \mathbf{M}^{s t}(r) \cdot \mathbf{K}_{M}^{s}(r) r^{2} \mathrm{dr}+\operatorname{dln} C^{s t} K_{C}^{s},
$$

where $\operatorname{dnn} \mathbf{M}^{s t}(r)$ represents the prescribed 3D $V_{S}, V_{P}$, and density heterogeneity at angular degree, $s$, order, $t$, and radius, $r$. $\mathbf{K}_{M}^{s}(r)$ are the relevant sensitivity kernels calculated using PREM ${ }^{79,60}$, $\operatorname{dn} \ln C^{s t}$ is the CMB topography (the discontinuity most important for Stoneley modes), and $K_{C}^{s}$ is the associated sensitivity kernel.

The misfit between predicted and observed Stoneley mode splitting functions, $\chi_{S}$ is

$$
\chi_{S}=\frac{1}{N_{S}} \sum_{n_{S}=1}^{N_{S}} \frac{\sum_{s=2}^{s_{\max }} \sum_{t=-s}^{s}\left(\mathcal{C}_{c}^{s t}-\mathcal{C}_{o}^{s t}\right)^{2}}{\sum_{s=2}^{s_{\max }} \sum_{t=-s}^{s}\left(\mathcal{C}_{o}^{s t}\right)^{2}}
$$

where $N_{S}=9$ is the number of individual Stoneley modes investigated, the second summation term includes only even degree terms, where $s_{\max }$ is the maximum order. In most calculations $s_{\max }=2$; however, we also test the impact of setting $s_{\max }$ to the maximum degree at which splitting function measurements are available for a particular mode, as well as the consequences of adopting different misfit criteria (Table S4). 
This preprint has been submitted to Nature Geoscience and is yet to undergo peer review.

We combine $\chi_{S}$ and $\chi_{G}$ to yield a joint total misfit function, $\chi_{T}$, using

$$
\chi_{T}=w_{G} \chi_{G}+w_{S} \chi_{S},
$$

where $w_{G}=0.5$ and $w_{S}=5$. These weightings result in misfit values with comparable global minima.

\section{References}

[1] Garnero, E. J., McNamara, A. K. \& Shim, S.-H. Continent-sized anomalous zones with low seismic velocity at the base of Earth's mantle. Nature Geoscience 9, 481-489 (2016).

[2] Hager, B. H., Clayton, R. W., Richards, M. A., Comer, R. P. \& Dziewonski, A. M. Lower mantle heterogeneity, dynamic topography and the geoid. Nature 313, 541-545 (1985).

[3] Ricard, Y., Richards, M., Lithgow-Bertelloni, C. \& Lestunff, Y. A Geodynamic Model of Mantle Density Heterogeneity. Journal of Geophysical Research: Solid Earth 98, 21895-21909 (1993).

[4] Cottaar, S. \& Lekic, V. Morphology of seismically slow lower-mantle structures. Geophysical Journal International 207, $1122-1136$ (2016).

[5] Koelemeijer, P., Schuberth, B., Davies, D., Deuss, A. \& Ritsema, J. Constraints on the presence of post-perovskite in Earth's lowermost mantle from tomographic-geodynamic model comparisons. Earth and Planetary Science Letters 494, 226-238 (2018).

[6] Maguire, R., Ritsema, J., Bonnin, M., van Keken, P. E. \& Goes, S. Evaluating the resolution of deep mantle plumes in teleseismic traveltime tomography. Journal of Geophysical Research: Solid Earth 123, 384-400 (2018).

[7] Davaille, A. \& Romanowicz, B. Deflating the LLSVPs: Bundles of Mantle Thermochemical Plumes Rather Than Thick Stagnant Piles. Tectonics 39, e2020TC006265 (2020).

[8] Grand, S. P. Mantle shear-wave tomography and the fate of subducted slabs. Philosophical Transactions of the Royal Society of London. Series A: Mathematical, Physical and Engineering Sciences 360, 2475-2491 (2002).

[9] Burke, K., Steinberger, B., Torsvik, T. H. \& Smethurst, M. A. Plume generation zones at the margins of large low shear velocity provinces on the core-mantle boundary. Earth and Planetary Science Letters 265, 49-60 (2008).

[10] Arevalo Jr, R. et al. Simplified mantle architecture and distribution of radiogenic power. Geochemistry, Geophysics, Geosystems 14, 2265-2285 (2013).

[11] Lu, C. et al. The Sensitivity of Joint Inversions of Seismic and Geodynamic Data to Mantle Viscosity. Geochemistry, Geophysics, Geosystems 21, e2019GC008648 (2020).

[12] Lau, H. C. et al. Tidal tomography constrains Earth's deep-mantle buoyancy. Nature 551, 321-326 (2017).

[13] Tackley, P. J. Dynamics and evolution of the deep mantle resulting from thermal, chemical, phase and melting effects. EarthScience Reviews 110, 1-25 (2012).

[14] Mulyukova, E., Steinberger, B., Dabrowski, M. \& Sobolev, S. V. Survival of LLSVPs for billions of years in a vigorously convecting mantle: Replenishment and destruction of chemical anomaly. Journal of Geophysical Research: Solid Earth 120, 3824-3847 (2015).

[15] Jones, T. D., Maguire, R. R., van Keken, P. E., Ritsema, J. \& Koelemeijer, P. Subducted oceanic crust as the origin of seismically slow lower-mantle structures. Progress in Earth and Planetary Science 7, 1-16 (2020). 
This preprint has been submitted to Nature Geoscience and is yet to undergo peer review.

[16] Moulik, P. \& Ekström, G. The relationships between large-scale variations in shear velocity, density, and compressional velocity in the Earth's mantle. Journal of Geophysical Research: Solid Earth 121, 2737-2771 (2016).

[17] Ni, S., Tan, E., Gurnis, M. \& Helmberger, D. Sharp sides to the African superplume. Science 296, 1850-1852 (2002).

[18] Schuberth, B. S. A., Bunge, H. P., Steinle-Neumann, G., Moder, C. \& Oeser, J. Thermal versus elastic heterogeneity in high-resolution mantle circulation models with pyrolite composition: High plume excess temperatures in the lowermost mantle. Geochem. Geophys. Geosyst. 10, Q01W01 (2009).

[19] Davies, D. R. et al. Reconciling dynamic and seismic models of Earth's lower mantle: The dominant role of thermal heterogeneity. Earth and Planetary Science Letters 353, 253-269 (2012).

[20] Ward, J., Nowacki, A. \& Rost, S. Lateral Velocity Gradients in the African Lower Mantle Inferred From Slowness Space Observations of Multipathing. Geochemistry, Geophysics, Geosystems 21, e2020GC009025 (2020).

[21] Ishii, M. \& Tromp, J. Even-degree lateral variations in the Earth's mantle constrained by free oscillations and the free-air gravity anomaly. Geophysical Journal International 145, 77-96 (2001).

[22] Koelemeijer, P., Deuss, A. \& Ritsema, J. Density structure of Earth's lowermost mantle from Stoneley mode splitting observations. Nature Communications 8, 1-10 (2017).

[23] Gurnis, M., Mitrovica, J. X., Ritsema, J. \& van Heijst, H.-J. Constraining mantle density structure using geological evidence of surface uplift rates: The case of the African Superplume. Geochem., Geophys., Geosyst. 1, $1999 \mathrm{GC000035} \mathrm{(2000).}$

[24] McNamara, A. K. \& Zhong, S. Thermochemical structures within a spherical mantle: Superplumes or piles? Journal of Geophysical Research: Solid Earth 109, B07402 (2004).

[25] Ghelichkhan, S. \& Bunge, H.-P. The adjoint equations for thermochemical compressible mantle convection: derivation and verification by twin experiments. Proceedings of the Royal Society A 474, 20180329 (2018).

[26] Dehant, V. et al. Understanding the effects of the core on the nutation of the Earth. Geodesy and Geodynamics 8, 389-395 (2017).

[27] Hoggard, M. J., White, N. \& Al-Attar, D. Global dynamic topography observations reveal limited influence of large-scale mantle flow. Nature Geoscience 9, 456-463 (2016).

[28] Chambat, F., Ricard, Y. \& Valette, B. Flattening of the Earth: further from hydrostaticity than previously estimated. Geophysical Journal International 183, 727-732 (2010).

[29] Hoggard, M. J., Winterbourne, J., Czarnota, K. \& White, N. Oceanic residual depth measurements, the plate cooling model, and global dynamic topography. Journal of Geophysical Research: Solid Earth 122, 2328-2372 (2017).

[30] Yang, T. \& Gurnis, M. Dynamic topography, gravity and the role of lateral viscosity variations from inversion of global mantle flow. Geophysical Journal International 207, 1186-1202 (2016).

[31] Watkins, C. E. \& Conrad, C. P. Constraints on dynamic topography from asymmetric subsidence of the mid-ocean ridges. Earth and Planetary Science Letters 484, 264-275 (2018).

[32] Davies, D. R. et al. Earth's multi-scale topographic response to global mantle flow. Nature Geoscience 12, 845-850 (2019).

[33] Steinberger, B., Conrad, C. P., Tutu, A. O. \& Hoggard, M. J. On the amplitude of dynamic topography at spherical harmonic degree two. Tectonophysics $\mathbf{7 6 0}, 221-228$ (2019).

[34] Koelemeijer, P. Towards consistent seismological models of the core-mantle boundary landscape. In Marquardt, H., Ballmer, M., Cottaar, S. \& Konter, J. G. (eds.) Mantle Convection and Surface Expressions, vol. 1 of Geophysical Monograph Series, 512 (Wiley Online Library, 2021). 
This preprint has been submitted to Nature Geoscience and is yet to undergo peer review.

[35] Richards, F. D., Hoggard, M. J., White, N. \& Ghelichkhan, S. Quantifying the relationship between short-wavelength dynamic topography and thermomechanical structure of the upper mantle using calibrated parameterization of anelasticity. Journal of Geophysical Research: Solid Earth 125, e2019JB019062 (2020).

[36] Deschamps, F., Cobden, L. \& Tackley, P. J. The primitive nature of large low shear-wave velocity provinces. Earth and Planetary Science Letters 349, 198-208 (2012).

[37] Valentine, A. P. \& Davies, D. R. Global models from sparse data: A robust estimate of Earth's residual topography spectrum. Geochemistry, Geophysics, Geosystems 21, e2020GC009240 (2020).

[38] Steinberger, B., Werner, S. C. \& Torsvik, T. H. Deep versus shallow origin of gravity anomalies, topography and volcanism on Earth, Venus and Mars. Icarus 207, 564-577 (2010).

[39] French, S. W. \& Romanowicz, B. Broad plumes rooted at the base of the Earth's mantle beneath major hotspots. Nature 525, 95-99 (2015)

[40] Forte, A. M. et al. Joint seismic-geodynamic-mineral physical modelling of African geodynamics: A reconciliation of deep-mantle convection with surface geophysical constraints. Earth and Planetary Science Letters 295, 329-341 (2010).

[41] Liu, X. \& Zhong, S. Constraining mantle viscosity structure for a thermochemical mantle using the geoid observation. Geochemistry, Geophysics, Geosystems 17, 895-913 (2016).

[42] Simmons, N., Myers, S., Johannesson, G., Matzel, E. \& Grand, S. Evidence for long-lived subduction of an ancient tectonic plate beneath the southern Indian Ocean. Geophysical Research Letters 42, 9270-9278 (2015).

[43] Niu, Y. Origin of the LLSVPs at the base of the mantle is a consequence of plate tectonics A petrological and geochemical perspective. Geoscience Frontiers 9, 1265-1278 (2018).

[44] Labrosse, S., Hernlund, J. \& Coltice, N. A crystallizing dense magma ocean at the base of the Earth's mantle. Nature 450, 866-869 (2007).

[45] Tolstikhin, I. \& Hofmann, A. W. Early crust on top of the Earth's core. Physics of the Earth and Planetary Interiors 148, 109-130 (2005).

[46] Lee, C.-T. A. et al. Upside-down differentiation and generation of a 'primordial' lower mantle. Nature 463, 930-933 (2010).

[47] Workman, R. K. \& Hart, S. R. Major and trace element composition of the depleted MORB mantle (DMM). Earth and Planetary Science Letters 231, 53-72 (2005).

[48] Connolly, J. A. D. Computation of phase equilibria by linear programming: A tool for geodynamic modeling and its application to subduction zone decarbonation. Earth and Planetary Science Letters 236, 524-541 (2005).

[49] Jones, T. D., Davies, D. R. \& Sossi, P. Tungsten isotopes in mantle plumes: Heads it's positive, tails it's negative. Earth and Planetary Science Letters 506, 255-267 (2019).

[50] Su, W.-J. \& Dziewonski, A. M. Simultaneous inversion for 3-D variations in shear and bulk velocity in the mantle. Physics of the Earth and Planetary Interiors 100, 135-156 (1997).

[51] Hoggard, M. J. et al. Global distribution of sediment-hosted metals controlled by craton edge stability. Nature Geoscience 13, $504-510(2020)$.

[52] Schaeffer, A. J. \& Lebedev, S. Global shear speed structure of the upper mantle and transition zone. Geophysical Journal International 194, 417-449 (2013).

[53] Schaeffer, A. J. \& Lebedev, S. Imaging the North American continent using waveform inversion of global and USArray data. Earth and Planetary Science Letters 402, 26-41 (2014). 
This preprint has been submitted to Nature Geoscience and is yet to undergo peer review.

[54] Celli, N. L., Lebedev, S., Schaeffer, A. J., Ravenna, M. \& Gaina, C. The upper mantle beneath the South Atlantic Ocean, South America and Africa from waveform tomography with massive data sets. Geophysical Journal International 221, 178-204 (2020).

[55] Celli, N. L., Lebedev, S., Schaeffer, A. J. \& Gaina, C. African cratonic lithosphere carved by mantle plumes. Nature Communications 11, 1-10 (2020).

[56] Jordan, T. H. Composition and development of the continental tectosphere. Nature 274, 544-548 (1978).

[57] Shapiro, S. S., Hager, B. H. \& Jordan, T. H. The continental tectosphere and Earth's long-wavelength gravity field. Lithos 48, $135-152(1999)$.

[58] Ritsema, J., Deuss, A., Van Heijst, H. J. \& Woodhouse, J. H. S40RTS: A degree-40 shear-velocity model for the mantle from new Rayleigh wave dispersion, teleseismic traveltime and normal-mode splitting function measurements. Geophysical Journal International 184, 1223-1236 (2011).

[59] Auer, L., Boschi, L., Becker, T., Nissen-Meyer, T. \& Giardini, D. Savani: A variable resolution whole-mantle model of anisotropic shear velocity variations based on multiple data sets. Journal of Geophysical Research: Solid Earth 119, 3006-3034 (2014).

[60] Dziewonski, A. M. \& Anderson, D. L. Preliminary reference Earth model. Physics of the Earth and Planetary Interiors 25, 297-356 (1981).

[61] Stixrude, L. \& Lithgow-Bertelloni, C. Thermodynamics of mantle minerals-II. Phase equilibria. Geophysical Journal International 184, 1180-1213 (2011).

[62] Matas, J. \& Bukowinski, M. S. On the anelastic contribution to the temperature dependence of lower mantle seismic velocities. Earth and Planetary Science Letters 259, 51-65 (2007).

[63] Hager, B. H. \& O'Connell, R. J. Kinematic models of large-scale flow in the Earth's mantle. Journal of Geophysical Research 84, $1031-1048(1979)$.

[64] Richards, M. A. \& Hager, B. H. Geoid anomalies in a dynamic Earth. Journal of Geophysical Research: Solid Earth 89, 5987-6002 (1984).

[65] Corrieu, V., Thoraval, C. \& Ricard, Y. Mantle dynamics and geoid Green functions. Geophysical Journal International 120, $516-523$ (1995).

[66] Moucha, R., Forte, A., Mitrovica, J. \& Daradich, A. Lateral variations in mantle rheology: implications for convection related surface observables and inferred viscosity models. Geophysical Journal International 169, 113-135 (2007).

[67] Ghosh, A., Becker, T. \& Zhong, S. Effects of lateral viscosity variations on the geoid. Geophysical Research Letters 37, L01301 (2010).

[68] Steinberger, B. \& Calderwood, A. R. Models of large-scale viscous flow in the Earth's mantle with constraints from mineral physics and surface observations. Geophysical Journal International 167, 1461-1481 (2006).

[69] Simmons, N. A., Forte, A. M. \& Grand, S. P. Joint seismic, geodynamic and mineral physical constraints on three-dimensional mantle heterogeneity: Implications for the relative importance of thermal versus compositional heterogeneity. Geophysical Journal International 177, 1284-1304 (2009).

[70] Steinberger, B. \& Holme, R. Mantle flow models with core-mantle boundary constraints and chemical heterogeneities in the lowermost mantle. Journal of Geophysical Research: Solid Earth 113, B05403 (2008).

[71] Gwinn, C. R., Herring, T. A. \& Shapiro, I. I. Geodesy by radio interferometry: Studies of the forced nutations of the Earth: 2. Interpretation. Journal of Geophysical Research: Solid Earth 91, 4755-4765 (1986). 
This preprint has been submitted to Nature Geoscience and is yet to undergo peer review.

[72] Mathews, P. M., Herring, T. A. \& Buffett, B. A. Modeling of nutation and precession: New nutation series for nonrigid Earth and insights into the Earth's interior. Journal of Geophysical Research: Solid Earth 107, ETG-3 (2002).

[73] Fei, H. et al. A nearly water-saturated mantle transition zone inferred from mineral viscosity. Science Advances 3, e1603024 (2017).

[74] Karato, S.-I. Importance of anelasticity in the interpretation of seismic tomography. Geophysical Research Letters 20, 1623-1626 (1993).

[75] Widmer, R., Masters, G. \& Gilbert, F. Observably split multiplets-data analysis and interpretation in terms of large-scale aspherical structure. Geophysical Journal International 111, 559-576 (1992).

[76] Lau, H. C. et al. A normal mode treatment of semi-diurnal body tides on an aspherical, rotating and anelastic Earth. Geophysical Journal International 202, 1392-1406 (2015).

[77] Resovsky, J. S. \& Ritzwoller, M. H. New and refined constraints on three-dimensional Earth structure from normal modes below $3 \mathrm{mHz}$. Journal of Geophysical Research: Solid Earth 103, 783-810 (1998).

[78] Ritsema, J., van Heijst, H. J. \& Woodhouse, J. H. Global transition zone tomography. Journal of Geophysical Research: Solid Earth 109, B02302 (2004).

[79] Woodhouse, J. The coupling and attenuation of nearly resonant multiplets in the Earth's free oscillation spectrum. Geophysical Journal International 61, 261-283 (1980). 